\title{
Partial Differential Operator on Vector Space of Polynomials
}

\author{
Chriestie E. J. C. Montolalu ${ }^{1}$ \\ ${ }^{1}$ Program Studi Matematika, FMIPA, UNSRAT Manado, chriestelly@yahoo.com
}

\begin{abstract}
A differential operator which acts on partial differentiation is defined as Partial Differential Operator (PDO). PDO works based on the order of the differential equation which then can solve the eigenvalues of the operator. On vector space of polynomials, PDO can be written in matrix representation. This can be helpful in finding the general form of eigenvalues of vector space polynomials. On this paper, a second order PDO:$$
D=\lambda\left(x_{1}^{2} \frac{\partial^{2}}{\partial x_{1}^{2}}+x_{2}^{2} \frac{\partial^{2}}{\partial x_{2}^{2}}\right)+\mu\left(x_{1} \frac{\partial}{\partial x_{2}}+x_{2} \frac{\partial}{\partial x_{1}}\right)
$$

will be operated on two and three variable vector space polynomials.

Keywords: differential equation, differential operator, eigenvalues, partial differential operator, vector space polynomials

\section{Operator Differensial Parsial pada Polinomial Vektor Ruang}

\begin{abstract}
Abstrak
Operator differensial yang dioperasikan pada differensial parsial didefinisikan sebagai Operator Differensial Parsial/Partial Differential Operator (PDO). PDO beroperasi mengacu pada pangkat tertinggi dari persamaan differensial yang kemudian bisa digunakan untuk menentukan nilai eigen dari operator tersebut. Pada polinomial vektor ruang, PDO bisa dituliskan dalam bentuk matriks. Hal ini bisa memudahkan dalam menentukan bentuk umum nilai eigen dari polinomial vektor ruang. Dalam tulisan ini,
\end{abstract} PDO orde dua:

$$
D=\lambda\left(x_{1}^{2} \frac{\partial^{2}}{\partial x_{1}^{2}}+x_{2}^{2} \frac{\partial^{2}}{\partial x_{2}^{2}}\right)+\mu\left(x_{1} \frac{\partial}{\partial x_{2}}+x_{2} \frac{\partial}{\partial x_{1}}\right)
$$

Akan dioperasikan pada polinomial vektor ruang dengan dua dan tiga variabel.

Kata kunci: persamaan differensial, operator differensial, nilai eigen, operator differensial parsial, polinomial vektor ruang

\section{Introduction}

A differential operator is an operator defined as a function of the differentiation operator [1]. If the operator acts on partial differentiation which includes more than one variable, then it can be defined as a Partial Differential Operator (PDO). A PDO, for example denoted by $D$, is linear if for any functions $u$ and $v$ and scalar $c$,

If $D$ is a linear PDO, the equation

$$
D[u+c v]=D u+c D v
$$

$$
D u=f
$$

is homogenous if $f \equiv 0$, and inhomogenous otherwise. The order of a partial differential equation is the order of the highest derivative appearing in it.

Based on the corresponding equation, the eigenvalue of the operator can be determined. In analogy to the matrix eigenvalue problem $A x=\lambda x$, we shall consider the eigenvalue problem

$$
D u=\mu u
$$

where $\mu$ is a real or complex number and $u$ is a non-zero function (i.e., $u$ is not identically equal to zero). $\mu$ will be called an eigenvalue of $D$ and $u$ is corresponding eigenvector.

On vector space of polynomials, PDO can be written in matrix representation [2]. For example, let $P_{3}$ be the vector space of polynomials of degree 3 and differential operator $D=\frac{\partial}{\partial x}$. The standard basis for this space is: $\left\{1, x, x^{2}, x^{3}\right\}$ which in vector notation is represented as: 


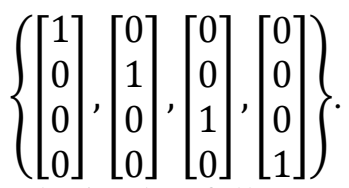

The differentiation of this space can be obtained as follows,

$D(1)=0$ corresponds to vector $\left[\begin{array}{llll}0 & 0 & 0 & 0\end{array}\right]$

$D(x)=1$ corresponds to vector $\left[\begin{array}{llll}1 & 0 & 0 & 0\end{array}\right]$

$D\left(x^{2}\right)=2 x$ corresponds to vector $\left[\begin{array}{llll}0 & 2 & 0 & 0\end{array}\right]$

$D\left(x^{3}\right)=3 x^{2}$ corresponds to vector $\left[\begin{array}{llll}0 & 0 & 3 & 0\end{array}\right]$

In matrix representation:

On this report, second order PDO:

$$
D=\left[\begin{array}{llll}
0 & 1 & 0 & 0 \\
0 & 0 & 2 & 0 \\
0 & 0 & 0 & 3 \\
0 & 0 & 0 & 0
\end{array}\right] .
$$

$$
D=\lambda\left(x_{1}^{2} \frac{\partial^{2}}{\partial x_{1}^{2}}+x_{2}^{2} \frac{\partial^{2}}{\partial x_{2}^{2}}\right)+\mu\left(x_{1} \frac{\partial}{\partial x_{2}}+x_{2} \frac{\partial}{\partial x_{1}}\right)
$$

will be operated on vector space of two-variable polynomials:

$$
V=\operatorname{span}\left\{x_{1}^{N-j} x_{2}^{j}, j=0, \ldots, N\right\} ; \operatorname{dim}\left(V^{N}\right)=N+1
$$

and a bit overview on vector space of three-variable polynomials:

$$
V=\operatorname{span}\left\{x_{1}^{j} x_{2}^{k} x_{3}^{N-j-k}, j=0, \ldots, N ; k=0, \ldots, N-j\right\}:
$$

Since $D$ in this case is a second order PDO, the corresponding partial differential equation will be in the form of second order partial differential equation. Subsequently, the eigenvalues of $D$ can be obtained from the equation by setting it into form (3).

\section{Partial Differential Operator On Two-Variable Polynomials}

Recall the differential operator used is (1.4) :

$$
D=\lambda\left(x_{1}^{2} \frac{\partial^{2}}{\partial x_{1}^{2}}+x_{2}^{2} \frac{\partial^{2}}{\partial x_{2}^{2}}\right)+\mu\left(x_{1} \frac{\partial}{\partial x_{2}}+x_{2} \frac{\partial}{\partial x_{1}}\right)
$$

and two-variable polynomials (1.5) :

$$
V=\operatorname{span}\left\{x_{1}^{N-j} x_{2}^{j}, j=0, \ldots, N\right\} ; \operatorname{dim}\left(V^{N}\right)=N+1
$$

e.g. $N=4$

Then the basis: $B=\left\{x_{1}^{4}, x_{1}^{3} x_{2}, x_{1}^{2} x_{2}^{2}, x_{1} x_{2}^{3}, x_{2}^{4}\right\}$

Thus,

$D\left(x_{1}^{4}\right)=12 \lambda x_{1}^{4}+4 \mu x_{1}^{3} x_{2}$

$D\left(x_{1}^{3} x_{2}\right)=\mu x_{1}^{4}+6 \lambda x_{1}^{3} x_{2}+3 \mu x_{1}^{2} x_{2}^{2}$

$D\left(x_{1}^{2} x_{2}^{2}\right)=2 \mu x_{1}^{3} x_{2}+4 \lambda x_{1}^{2} x_{2}^{2}+2 \mu x_{1}^{2} x_{2}^{2}$

$D\left(x_{1} x_{2}^{3}\right)=3 \mu x_{1}^{2} x_{2}^{2}+6 \lambda x_{1} x_{2}^{3}+\mu x_{2}^{4}$

$D\left(x_{2}^{4}\right)=4 \mu x_{1} x_{2}^{3}+12 \lambda x_{2}^{4}$

Therefore,

$$
D=\left(\begin{array}{ccccc}
12 \lambda & \mu & 0 & 0 & 0 \\
4 \mu & 6 \lambda & 2 \mu & 0 & 0 \\
0 & 3 \mu & 4 \lambda & 3 \mu & 0 \\
0 & 0 & 2 \mu & 6 \lambda & 4 \mu \\
0 & 0 & 0 & \mu & 12 \lambda
\end{array}\right)
$$

In general,

$D\left(x_{1}^{N-j} x_{2}^{j}\right)=A x_{1}^{N-j} x_{2}^{j}+B x_{1}^{N-j+1} x_{2}^{j-1}+C x_{1}^{N-j} x_{2}^{j+1}$

where,

$A=\lambda[(N-j)(N-j-1)+j(j-1)]$

$B=\mu j$

$C=\mu(N-j)$ 
In matrix representation:

$$
\begin{aligned}
& A=\left(\begin{array}{ccccc}
\lambda(N)(N-1) & 0 & \ldots & 0 & 0 \\
0 & \lambda[(N-1)(N-2)] & \ldots & 0 & 0 \\
\vdots & \vdots & \ddots & \vdots & \vdots \\
0 & 0 & \ldots & \lambda[(N-1)(N-2)] & 0 \\
0 & 0 & \ldots & 0 & \lambda[N(N-1)]
\end{array}\right) \\
& B=\left(\begin{array}{cccccc}
0 & \mu & 0 & \cdots & 0 & 0 \\
0 & 0 & 2 \mu & \cdots & 0 & 0 \\
0 & 0 & 0 & \cdots & 0 & 0 \\
\vdots & \vdots & \vdots & \ddots & (N-1) \mu & \vdots \\
0 & 0 & 0 & \cdots & 0 & N \mu \\
0 & 0 & 0 & \cdots & 0 & 0
\end{array}\right) \\
& C=\left(\begin{array}{cccccc}
0 & 0 & \ldots & 0 & 0 & 0 \\
N \mu & 0 & \ldots & 0 & 0 & 0 \\
0 & (N-1) \mu & \ldots & 0 & 0 & 0 \\
\vdots & \vdots & \ddots & \vdots & \vdots & \vdots \\
0 & 0 & \ldots & 2 \mu & 0 & 0 \\
0 & 0 & \ldots & 0 & \mu & 0
\end{array}\right)
\end{aligned}
$$

\subsection{Differential Equation}

The corresponding Partial Differential Equation can be obtained as follows:

Suppose:

$$
\begin{gathered}
w^{j}=x_{1}^{N-j} x_{2}^{j} \\
\text { and } \\
Q(w)=\prod_{j=1}^{N}\left(w-v_{j}\right)
\end{gathered}
$$

Thus,

$\frac{d w^{j}}{d w}=j w^{j-1}=j x_{1}^{N-j+1} x_{2}^{j-1}$

and

$w\left(w^{j}\right)=w^{j+1}=x_{1}^{N-j-1} x_{2}^{j+1}$

The differential equation can be written as follows:

where

$$
\tilde{A}(w) Q^{\prime \prime}(w)+\tilde{B}(w) Q^{\prime}(w)+\tilde{C}(w) Q(w)=E Q(w)
$$

$\tilde{A}(w)=2 \lambda w^{2}$

$\tilde{B}(w)=-2 \lambda w(N-1)+\mu\left(1-w^{2}\right)$

$\tilde{C}(w)=\lambda N(N-1)+\mu N w$

and $E$ as eigenvalues of $D$

Proof

$$
\begin{aligned}
A\left(w^{j}\right) & =\lambda[(N-j)(N-j-1)+j(j-1)] w^{j} \\
& =\lambda\left[N(N-1)-2 N j+2 j^{2}\right] w^{j} \\
& =\lambda\left[N(N-1) w^{j}-2 N w \frac{d w^{j}}{d w}+2\left(w^{2} \frac{d^{2} w^{j}}{d w^{2}}+w \frac{d w^{j}}{d w}\right)\right] \\
& =\lambda\left[N(N-1) w^{j}+(2 w-2 N w) \frac{d w^{j}}{d w}+2 w^{2} \frac{d^{2} w^{j}}{d w^{2}}\right] \\
B\left(w^{j}\right) & =\mu j w^{j-1}=\mu \frac{d w^{j}}{d w} \\
C\left(w^{j}\right) & =\mu(N-j) w^{j+1}=\mu N w \cdot w^{j}-\mu w^{2} \frac{d w^{j}}{d w}
\end{aligned}
$$

The coefficient of $Q^{\prime \prime}(w)$ is taken from the coefficient of $\frac{d^{2} w^{j}}{d w^{2}}$, thus $\tilde{A}(w)=2 \lambda w^{2}$

The coefficient of $Q^{\prime}(w)$ is taken from the coefficient of $\frac{d w^{j}}{d w}$, thus 
$\widetilde{B}(w)=-2 \lambda w(N-1)+\mu\left(1-w^{2}\right)$

The coefficient of $Q(w)$ is taken from the coefficient of $w^{j}$, thus

$\tilde{C}(w)=\lambda N(N-1)+\mu N w$

\section{Theorem 1}

The quotient of the first and second derivatives of (8) can be obtained as:

\section{Proof}

$$
\frac{Q^{\prime \prime}\left(v_{k}\right)}{Q^{\prime}\left(v_{k}\right)}=-\sum_{\substack{j=1 \\ k \neq j}}^{N} \frac{2}{\left(v_{k}-v_{j}\right)}
$$

It is clear that the first and second derivatives of $Q(w)=\prod_{j=1}^{N}\left(w-v_{j}\right)$ can be obtained as follows,

$Q^{\prime}(w)=Q(w) \sum_{j=1}^{N} \frac{1}{\left(w-v_{j}\right)}$

and

$$
\begin{aligned}
Q^{\prime \prime}(w) & =\sum_{j=1}^{N} \frac{Q^{\prime}(w)\left(w-v_{j}\right)-Q(w)}{\left(w-v_{j}\right)^{2}} \\
& =Q^{\prime}(w) \sum_{j=1}^{N} \frac{\left(w-v_{j}\right)-\frac{1}{\sum_{j=1}^{N} \frac{1}{\left(w-v_{j}\right)}}}{\left(w-v_{j}\right)^{2}} \\
& =Q^{\prime}(w)\left[\sum_{j=1}^{N} \frac{1}{w-v_{j}}-\sum_{j=1}^{N} \frac{\frac{Q(w)}{\sum_{j=1}^{N} \frac{Q(w)}{\left(w-v_{j}\right)}}}{\left(w-v_{j}\right)^{2}}\right]
\end{aligned}
$$

Suppose $w \rightarrow v_{k}$ such that $k \neq j$, thus

$$
\lim _{w \rightarrow v_{k}} \frac{Q^{\prime \prime}(w)}{Q^{\prime}(w)}=-\sum_{\substack{j=1 \\ k \neq j}}^{N} \frac{2}{\left(v_{k}-v_{j}\right)}
$$

\subsection{Eigenvalues}

From (9), the eigenvalues can be obtained as:

\section{Proof}

$$
E=-\mu\left(\sum_{j=1}^{N} v_{j}\right)+\lambda N(N-1)
$$

The asymptotic expansion of $Q(w)$ can be written as follows:

$Q(w) \sim w^{N}-\left(\sum_{j=1}^{N} v_{j}\right) w^{N-1}+\cdots$

Then, the first and second derivative of $Q(w)$ can be written as:

$Q^{\prime}(w) \sim N w^{N-1}-(N-1)\left(\sum_{j=1}^{N} v_{j}\right) w^{N-2}+\cdots$

$Q^{\prime \prime}(w) \sim N(N-1) w^{N-2}-(N-1)(N-2)\left(\sum_{j=1}^{N} v_{j}\right) w^{N-3}+\cdots$

Substitute these into (2.3), hence

$$
E Q(w)=\left[-\mu\left(\sum_{j=1}^{N} v_{j}\right)+\lambda N(N-1)\right] w^{N}+O\left(w^{N-1}\right)
$$

\subsection{Symmetric and Anti-Symmetric Basis}

Rather than just the regular basis analyzed above, other different basis can be used to obtain various results. In this case, symmetric and anti-symmetric basis are used and considered to be as follows:

$B^{\prime}=B_{+}^{\prime} \cup B_{-}^{\prime}$

with 
$B_{+}^{\prime}=\left\{x_{1}^{N}+x_{2}^{N}, x_{1}^{N-1} x_{2}+x_{1} x_{2}^{N-1}, \ldots, x_{1}^{N-j} x_{2}^{j}+x_{1}^{j} x_{2}^{N-j}\right\}$ as symmetric basis, and $B_{-}^{\prime}=\left\{x_{1}^{N-j} x_{2}^{j}-x_{1}^{j} x_{2}^{N-j}, \ldots, x_{1}^{N-1} x_{2}-x_{1} x_{2}^{N-1}, x_{1}^{N}-x_{2}^{N}\right\}$ as anti-symmetric basis.

e.g. for $N=4$

$B^{\prime}=B_{+}^{\prime} \cup B_{-}^{\prime}$

where,

$B_{+}^{\prime}=\left\{x_{1}^{4}+x_{2}^{4}, x_{1}^{3} x_{2}+x_{1} x_{2}^{3}, x_{1}^{2} x_{2}^{2}\right\}$

$B_{-}^{\prime}=\left\{x_{1}^{3} x_{2}-x_{1} x_{2}^{3}, x_{1}^{4}-x_{2}^{4}\right\}$

Thus,

$$
\begin{aligned}
& D\left(x_{1}^{4}+x_{2}^{4}\right)=12 \lambda\left(x_{1}^{4}+x_{2}^{4}\right)+4 \mu\left(x_{1}^{3} x_{2}+x_{1} x_{2}^{3}\right) \\
& D\left(x_{1}^{3} x_{2}+x_{1} x_{2}^{3}\right)=\mu\left(x_{1}^{4}+x_{2}^{4}\right)+6 \lambda\left(x_{1}^{3} x_{2}+x_{1} x_{2}^{3}\right)+6 \mu x_{1}^{2} x_{2}^{2} \\
& D\left(x_{1}^{2} x_{2}^{2}\right)=2 \mu\left(x_{1}^{3} x_{2}+x_{1} x_{2}^{3}\right)+4 \lambda x_{1}^{2} x_{2}^{2} \\
& D\left(x_{1}^{3} x_{2}-x_{1} x_{2}^{3}\right)=6 \lambda\left(x_{1}^{3} x_{2}-x_{1} x_{2}^{3}\right)+\mu\left(x_{1}^{4}-x_{2}^{4}\right) \\
& D\left(x_{1}^{4}-x_{2}^{4}\right)=4 \mu\left(x_{1}^{3} x_{2}-x_{1} x_{2}^{3}\right)+12 \lambda\left(x_{1}^{4}-x_{2}^{4}\right)
\end{aligned}
$$

Therefore,

$$
D=\left(\begin{array}{ccccc}
12 \lambda & \mu & 0 & 0 & 0 \\
4 \mu & 6 \lambda & 2 \mu & 0 & 0 \\
0 & 6 \mu & 4 \lambda & 0 & 0 \\
0 & 0 & 0 & 6 \lambda & 4 \mu \\
0 & 0 & 0 & \mu & 12 \lambda
\end{array}\right)
$$

In general,

$$
\begin{gathered}
B^{\prime}=\left\{\begin{array}{c}
x_{1}^{N}+x_{2}^{N}, x_{1}^{N-1} x_{2}+x_{1} x_{2}^{N-1}, \ldots, x_{1}^{N-j} x_{2}^{j}+x_{1}^{j} x_{2}^{N-j}, \\
x_{1}^{N-j} x_{2}^{j}-x_{1}^{j} x_{2}^{N-j}, \ldots, x_{1}^{N-1} x_{2}-x_{1} x_{2}^{N-1}, x_{1}^{N}-x_{2}^{N}
\end{array}\right\} \\
\begin{aligned}
D\left(x_{1}^{N-j} x_{2}^{j}+x_{1}^{j} x_{2}^{N-j}\right)= & A\left(x_{1}^{N-j} x_{2}^{j}+x_{1}^{j} x_{2}^{N-j}\right)+ \\
& B\left(x_{1}^{N-j+1} x_{2}^{j-1}+x_{1}^{j-1} x_{2}^{N-j+1}\right)+ \\
& C\left(x_{1}^{N-j-1} x_{2}^{j+1}+x_{1}^{j+1} x_{2}^{N-j-1}\right)
\end{aligned}
\end{gathered}
$$

and

$$
\begin{aligned}
D\left(x_{1}^{N-j} x_{2}^{j}-x_{1}^{j} x_{2}^{N-j}\right)= & X\left(x_{1}^{N-j} x_{2}^{j}-x_{1}^{j} x_{2}^{N-j}\right)+ \\
& Y\left(x_{1}^{N-j+1} x_{2}^{j-1}-x_{1}^{j-1} x_{2}^{N-j+1}\right)+ \\
& Z\left(x_{1}^{N-j-1} x_{2}^{j+1}-x_{1}^{j+1} x_{2}^{N-j-1}\right)
\end{aligned}
$$

where,

$$
\begin{aligned}
& A=X=\lambda[(N-j)(N-j-1)+j(j-1)] \\
& B=Y=\mu j \\
& Z=\mu(N-j)
\end{aligned}
$$

In matrix representation:

$$
D=\left(\begin{array}{cc}
A+B+C & 0 \\
0 & X+Y+Z
\end{array}\right)
$$

Unfortunately, the general form of terms in matrix $C$ cannot be easily determined and solved in this paper. An in-depth analysis is needed to determine it. Nevertheless, it can be done in the future research.

Even though the general terms are still not completed here, the eigenvalues will be able to be determined once the general terms found. As it is in the simple basis above, the steps to determine the eigenvalues can be done as follows,

Suppose: $w^{j}=x_{1}^{N-j} x_{2}^{j}+x_{1}^{j} x_{2}^{N-j}$

Then,

$\frac{d w^{j}}{d w}=j w^{j-1}=j\left(x_{1}^{N-j+1} x_{2}^{j-1}+x_{1}^{j-1} x_{2}^{N-j+1}\right)$ 
$w\left(w^{j}\right)=w^{j+1}=x_{1}^{N-j-1} x_{2}^{j+1}+x_{1}^{j+1} x_{2}^{N-j+1}$

$A Q^{\prime \prime}(w)+B Q^{\prime}(w)+C Q(w)=E_{1} Q(w)$

and

$X(w)+Y Q^{\prime}(w)+Z Q(w)=E_{2} Q(w)$

where $E_{1}$ and $E_{2}=$ eigenvalues

\section{Partial Differential Operator on Three-Variable Polynomials}

Differential operator for three-variable polynomials :

$$
\begin{gathered}
D=\lambda\left(x_{1}^{2} \frac{\partial^{2}}{\partial x_{1}^{2}}+x_{2}^{2} \frac{\partial^{2}}{\partial x_{2}^{2}}+x_{3}^{2} \frac{\partial^{2}}{\partial x_{3}^{2}}\right)+ \\
\mu\left(x_{1} \frac{\partial}{\partial x_{2}}+x_{1} \frac{\partial}{\partial x_{3}}+x_{2} \frac{\partial}{\partial x_{1}}+x_{2} \frac{\partial}{\partial x_{3}}+x_{3} \frac{\partial}{\partial x_{1}}+x_{3} \frac{\partial}{\partial x_{2}}\right)
\end{gathered}
$$

and recall three-variable polynomials (6):

e.g. $N=2$

$$
V=\operatorname{span}\left\{x_{1}^{j} x_{2}^{k} x_{3}^{N-j-k}, j=0, \ldots, N ; k=0, \ldots, N-j\right\}
$$

$V^{2}=\left\{x_{1}^{2}, x_{2}^{2}, x_{3}^{2}, x_{1} x_{2}, x_{1} x_{3}, x_{2} x_{3}\right\}$

$D$ can be obtained as:

$$
D=\left[\begin{array}{cccccc}
2 \lambda & 0 & 0 & \mu & \mu & 0 \\
0 & 2 \lambda & 0 & \mu & 0 & \mu \\
0 & 0 & 2 \lambda & 0 & \mu & \mu \\
2 \mu & 2 \mu & 0 & 0 & \mu & \mu \\
2 \mu & 0 & 2 \mu & \mu & 0 & \mu \\
0 & 2 \mu & 2 \mu & \mu & \mu & 0
\end{array}\right]
$$

General forms for this are still in development, nevertheless the theory behind it can be based on two-variable polynomials case.

\section{References}

[1] Yakubov, S. and Y. Yakubov, 2000. Differential-Operator Equations: Ordinary and Partial Differential Equations, Chapman and Hall/CRC, Florida.

[2] Lanczos, C., 1997. Linear Differential Operators, Dover Publications Inc., New York. 